Supporting Information

\title{
Hierarchical Mesoporous Iron Fluoride and Reduced Graphene oxide Nanocomposite as cathode Materials for High-Performance Sodium-Ion Batteries
}

Chao Zhang ${ }^{\dagger \S}$, Shengli An ${ }^{\dagger}$, Wenting Li ${ }^{\dagger}$, Hanying Xu, Weijian Hao ${ }^{\ddagger}$, Wei Liu ${ }^{\ddagger}$, Zelin $\mathrm{Li}^{\S}$, and Xinping Qiu*\$

${ }^{\dagger}$ School of Metallurgical and Ecological Engineering, University of Science and Technology Beijing, Beijing 100083, China

Key Laboratory of Organic Optoelectronics and Molecular Engineering, Department of Chemistry, Tsinghua University, Beijing 100084, China

${ }^{\S}$ Inner Mongolia University of Science and Technology, School of Materials and Metallurgy, Baotou 014010, China

The detailed calculation process of TG:

We assume that the mass of FFH-G nanocomposite is $1 \mathrm{~g}$ and the amount of $\mathrm{rGO}$ in the nanocomposite is $\mathrm{x}$.

$$
\begin{aligned}
& (1-x) \times 0.8015=0.7523 \\
& x=0.0614
\end{aligned}
$$

So it can be calculated that rGO comprises $6.14 \%$ of the nanocomposite mass based on the result. In the orther words, $\mathrm{FeF}_{3} \bullet 0.33 \mathrm{H}_{2} \mathrm{O}$ loading is $93.86 \%$. 

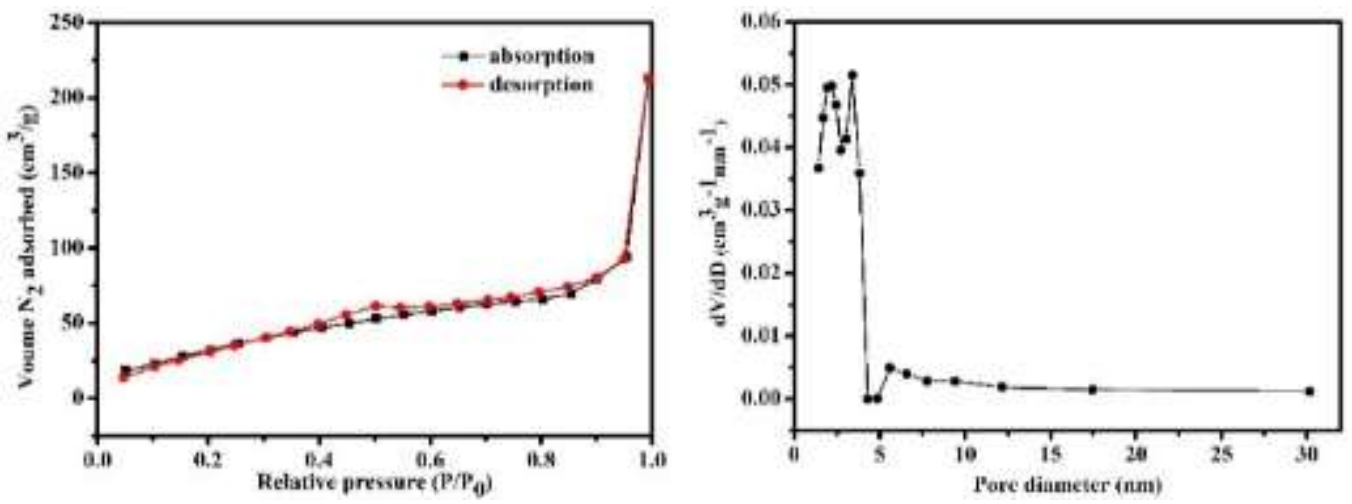

Figure S1. (a) $\mathrm{N}_{2}$ adsorption/desorption isotherms of FFH nanoparticle; (b) The BJH pore size distribution of FFH nanoparticle. 